\title{
Development and validation of a difficulty index for mandibular third molars with extraction time
}

\author{
Jeong-Kui Ku', Na-Hee Chang ${ }^{2}$, Yeong-Kon Jeong ${ }^{1}$, Sung Hyun Baik², Sun-Kyu Choi ${ }^{4}$ \\ ${ }^{1}$ Department of Oral and Maxillofacial Surgery, Section of Dentistry, Armed Forces Capital Dental Hospital, Armed Forces Medical \\ Command, Seongnam, ${ }^{2}$ Biomedical Research Institute, Seoul National University Bundang Hospital, Seongnam, \\ ${ }^{3}$ Department of Radiology, Seoul National University Bundang Hospital, Seongnam, \\ ${ }^{4}$ Department of Biostatistics, Korea University College of Medicine, Seoul, Korea
}

\begin{abstract}
J Korean Assoc Oral Maxillofac Surg 2020;46:328-334)
Objectives: This study aimed to validate the effectiveness of a recently proposed difficulty index for removal of impacted mandibular third molars based on extraction time and suggest a modified difficulty index including the presence of pathologic conditions associated with third molars.

Materials and Methods: This retrospective study enrolled 65 male patients younger than 25 years with third molars. Extraction time was calculated from start of the incision to the last suture. The difficulty scores for third molars were based on spatial relationship (1-5 points), depth (1-4 points), and ramus relationship (1-3 points) using cone-beam computed tomography. The difficulty index was defined as follows: I (3-4 points), II (5-7 points), III (8-10 points), and IV (11-12 points). The modified difficulty score was calculated by adding one point to the difficulty score if the third molar was associated with a pathologic condition. Two modified difficulty indices, based on the presence of pathologic conditions, were as follows: the half-level up difficulty index (HDI) and the one-level up difficulty index (ODI) from the recently proposed difficulty index.

Results: The correlations between extraction time and difficulty index and or modified difficulty indices were significant $(P<0.001)$. The correlation coefficient between extraction time and difficulty index was 0.584 . The correlation coefficients between extraction time and HDI and ODI were 0.728 and 0.764 , respectively.

Conclusion: Extraction time of impacted third molars exhibited a moderate correlation with difficulty index and was strongly correlated with the modified indices. Considering the clinical implications, the difficulty index of surgical extraction should take into consideration the pathologic conditions associated with third molars.
\end{abstract}

Key words: Computed tomography, Classification, Tooth extraction, Impacted tooth, Third molar

[paper submitted 2020. 2. 9 / revised 1st 2020. 3. 10, 2nd 2020. 3. 21 / accepted 2020. 3. 24]

\section{Introduction}

Surgical difficulty in mandibular third molar extractions can predict the incidence of postoperative complications. Since the 19th century, several researchers have attempted to classify extraction difficulty using radiographic techniques or

\footnotetext{
Jeong-Kui $\boldsymbol{K u}$

Department of Oral and Maxillofacial Surgery, Section of Dentistry, Armed Forces Capital Dental Hospital, Armed Forces Medical Command, 81 Saemaeul-ro 177beon-gil, Bundang-gu, Seongnam 13574, Korea

TEL: +82-31-725-6184 FAX: +82-31-706-0987

E-mail:kujk123@gmail.com

ORCID: https://orcid.org/0000-0003-1192-7066

(c) This is an open-access article distributed under the terms of the Creative Commons Attribution Non-Commercial License (http://creativecommons.org/ licenses/by-nc/4.0/), which permits unrestricted non-commercial use, distribution, and reproduction in any medium, provided the original work is properly cited.

Copyright (C) 2020 The Korean Association of Oral and Maxillofacial Surgeons. All rights reserved.
}

estimating the prevalence of complications ${ }^{1-5}$. In December 2019, a difficulty index was proposed based on positional variations in impacted mandibular third molars evaluated by panoramic radiography ${ }^{5}$. The position of the third molar is associated not only with postoperative complications, but also surgical difficulty and operation time $\mathrm{e}^{6,7}$. In the Korean insurance system, surgical time is one of the important criteria for evaluating surgical difficulty ${ }^{8}$. However, previous difficulty indices focused mainly on the probability of complications, and very few clinical studies have reported on prediction of surgery time $\mathrm{e}^{1-5}$.

Evaluating extraction time is difficult given the various surgical techniques used and the highly divergent morphology of third molars and their surrounding structures ${ }^{9}$. The operculum is an epithelial tissue overlying the impacted tooth that originates from the dental follicle and attaches to the ce- 
mentoenamel junction $(\mathrm{CEJ})^{10}$. The operculectomy procedure enables access to the CEJ for odontomy, facilitates surgical extraction through a minimally invasive technique, and enhances healing by de-epithelialization.

Impacted third molars may be associated with pathologic conditions such as root resorption of the adjacent tooth, pericoronitis, dentigerous cysts, and benign neoplasms ${ }^{11}$. However, few studies have evaluated the presence of pathologic conditions even though they are more frequently encountered than complications ${ }^{5}$.

The present study aimed to assess the validity of the difficulty index with extraction time using a unified incision technique (operculectomy) and suggests a modified difficulty index including the presence of pathologic conditions associated with third molars.

\section{Materials and Methods}

This retrospective study was initiated after approval by the Institutional Review Board at the Armed Forces Capital Hospital (No. AFCH-19-IRB-034). We enrolled healthy male patients who had undergone mandibular third molar extraction by one expert surgeon from December 2018 to December 2019 at the Armed Forces Capital Dental Hospital. The inclusion criteria were as follows: 1) age between 19 and 25 years, 2) preoperative computed tomography (CT) evaluation (syngo CT VB20, Sickness 1.0 mm, Dose 120 kVp; Siemens, Berlin, Germany), 3) surgical approach by operculectomy alone, 4) intraoperative digital photographs taken at the start of incision; after the incision; and during odontomy, extraction, and wound suturing.(Fig. 1) The exclusion criteria were as follows: 1) any incision design (vertical incision, envelope incision) except operculectomy, 2) a history or symptoms of

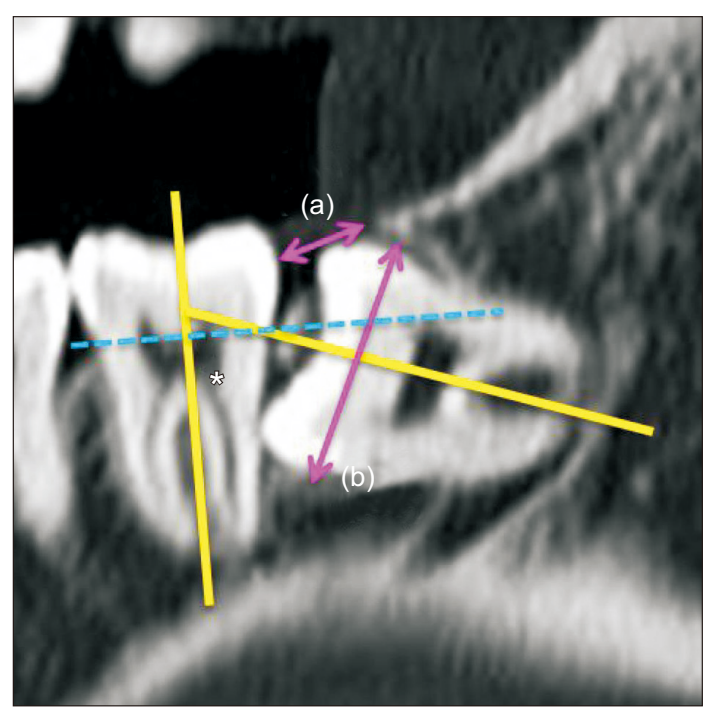

Fig. 2. Measurement and classification of the impacted third molars in cone-beam computed tomography according to a previous study conducted by Kim et al. ${ }^{5}$. The spatial relationship was classified based on the angle measured between the long axis of the impacted third molar and the adjacent second molar (yellow lines and an asterisk mark). Depth was classified based on the line connecting the cementoenamel junction of the adjacent second molar (dotted blue line). Ramus relationship/space available was subcategorized based on the ratio between the distance from the ascending ramus to the distal of the second molar (a) and the diameter of the impacted third molar (b) (pink arrows).

Jeong-Kui Ku et al: Development and validation of a difficulty index for mandibular third molars with extraction time. J Korean Assoc Oral Maxillofac Surg 2020
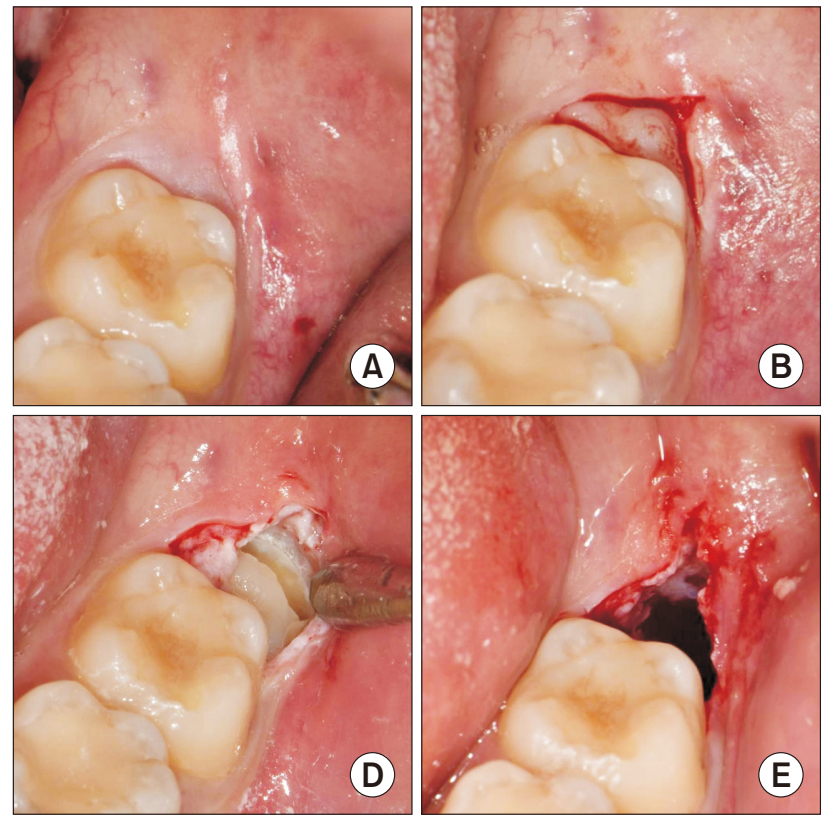

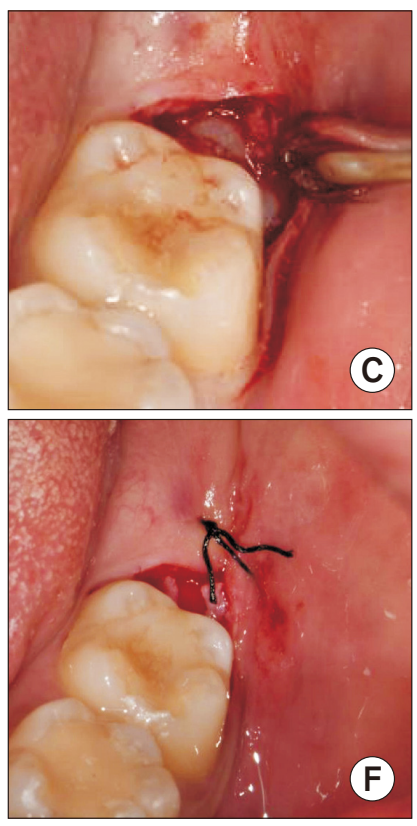

Fig. 1. Photographs of the surgical extractions with operculectomies. A. Preoperative photograph of the patient in Fig. 2. B. Incision for the operculectomy. C. After operculectomy, the impacted mandibular third molar was exposed. D. Odontomy of the third molar was performed. E. Extraction of the third molar was completed. F. Postoperative photograph after suturing with collagen plug packing.

Jeong-Kui Ku et al: Development and validation of a difficulty index for mandibular third molars with extraction time. J Korean Assoc Oral Maxillofac Surg 2020 
maxillary sinus disease, 3 ) poor plaque control and untreated chronic periodontitis, and 4) third molars with incomplete root formation.

The pathologic conditions associated with impacted third molars were defined as caries, pericoronitis, dentigerous cysts, deformed roots, cuspal contact below the CEJ of the adjacent molar, and root resorption of the adjacent tooth.

Extraction time was calculated from the start of the incision to the last suture, based on digital photographs.

\section{Difficulty score and index}

We reformatted the CT data to a panoramic view based on the mandibular arch (middle of the mandibular teeth and inferior mandibular canal) with an axial cut using platform software (SOMARIS/7; Siemens) and selected a single image cut that included the middle of the extracted third molar.

We followed the most recently published (2019) difficulty index ${ }^{5}$. Briefly, impacted third molars were classified and scored (difficulty score) based on spatial relationship (1-5 points), depth (1-4 points), and ramus relationship (1-3 points).(Fig. 2)

Spatial relationship was subcategorized based on the angle between the long axis of the third molar and the adjacent second molar as follows: (1) mesioangular $\left(11^{\circ}\right.$ to $\left.79^{\circ}\right)$, (2) horizontal $\left(80^{\circ}\right.$ to $\left.100^{\circ}\right)$, (3) vertical $\left(-10^{\circ}\right.$ to $\left.10^{\circ}\right)$, (4) distoangular $\left(-11^{\circ}\right.$ to $\left.-79^{\circ}\right)$, or $(5)$ reverse where the crown of the third molar was more root-oriented than horizontal.

Depth was subcategorized and scored as levels A (1), B (2), C (3), or D (4). Level A was defined as a condition when more than half of the third molar crown was above the CEJ of the adjacent second molar. Level B was defined when less than half of the third molar crown above the CEJ of the adjacent second molar. When the entire the third molar crown was positioned below the CEJ of the adjacent second molar, it was defined as either level C or level D. Level C was defined as a condition when more than half of the third molar crown was positioned superior to the mid-level of the adjacent second molar root. Level D was defined when the third molar crown level was inferior to that mentioned above.

The available ramus relationship/space was sub-classified and scored as class I (1), II (2), or III (3). Eruption space was defined as the ratio of distance between the distal side of the second molar to the ascending ramus (a) and the diameter of the third molar (b), as shown in Fig. 2. An eruption space (a/b) larger than two-thirds the distance was defined as class I, between one-third and two-thirds was class II, and smaller than one-third was class III.

The difficulty index was based on total points as follows: I (3-4 points), II (5-7 points), III (8-10 points), and IV (11-12 points). In addition, the modified difficulty score was calculated by adding one point to the difficulty score if the third molar was associated with a pathologic condition. Two modified difficulty indices that considered the presence of pathologic conditions were defined as follows: the half-level up difficulty index (HDI) and the one-level up difficulty index (ODI) based on the difficulty index reported by Kim et al. ${ }^{5}$.

\section{Statistical analysis}

Extraction time was analyzed with an independent t-test for difference between anesthetic techniques (general or local anesthesia) and left- or right-side molar. One-way ANOVA was used to analyze the difference in extraction time between difficulty indices. Post-hoc analysis was performed using the Mann-Whitney U test, and the Bonferroni method was used to control for type 1 error. Pearson correlation coefficients were used to reveal linear relationships of extraction time with difficulty score and index and with modified difficulty score and indices (HDI and ODI). The data were analyzed using IBM SPSS Statistics (ver. 23.0; IBM, Armonk, NY, USA). A $P$-value less than 0.05 indicated significance.

\section{Results}

A total of 65 patients (all males, 21.25 \pm 1.37 years) with 68 extractions was included, and the average extraction time was $17.48 \pm 6.56$ minutes. Fifty-one extractions were performed under local anesthesia (18.04 \pm 6.56 minutes), while 17 were completed under general anesthesia (17.48 \pm 6.56 minutes).

Table 1. Demographic and clinical informations of the patients with impacted third molar

\begin{tabular}{lcc}
\hline \multicolumn{1}{c}{ Variable } & Value & $P$-value ${ }^{1}$ \\
\hline No. of patients & 65 & \\
Age (yr) & $21.25 \pm 1.37$ & \\
Sex & Male & \\
Extraction time (min) & & \\
$\quad$ Anesthesia & & 0.439 \\
$\quad$ Local ( $\mathrm{n}=51)$ & $18.04 \pm 6.56$ & \\
$\quad$ General (n=17) & $17.48 \pm 6.56$ & \\
$\quad$ Location & & 0.959 \\
$\quad$ Left $(\mathrm{n}=34)$ & $18.17 \pm 6.58$ & \\
$\quad$ Right (n=34) & $16.55 \pm 7.11$ & \\
\hline
\end{tabular}

${ }^{1}$ Independent sample t-test.

Values are presented as mean \pm standard deviation.

Jeong-Kui Ku et al: Development and validation of a difficulty index for mandibular third molars with extraction time. J Korean Assoc Oral Maxillofac Surg 2020 
Thirty-four extractions were performed on the left side (18.17 \pm 6.58 minutes), and 34 were completed on the right side (16.55 \pm 7.11 minutes). Extraction time exhibited no statistical difference according to anesthetic technique or side. (Table 1)

The mean extraction times according to difficulty score were $9.47 \pm 4.33,15.08 \pm 5.40,18.13 \pm 5.36,17.28 \pm 4.20$, $19.51 \pm 6.42$, and $28.20 \pm 5.17$ minutes from scores three to eight, respectively. The correlation between extraction time and difficulty score was significant $(P<0.001)$, and the observed correlation coefficient (r) was 0.599 , suggesting a moderate positive correlation $(P<0.001)$. The mean extraction times according to modified difficulty scores were $8.59 \pm 4.10,14.30 \pm 3.01,15.41 \pm 4.59,19.08 \pm 5.07,17.18 \pm 6.06$, $26.09 \pm 3.32$, and $27.36 \pm 5.33$ minutes from scores three to nine, respectively. The correlation between extraction time and modified difficulty score was significant $(P<0.001)$. The observed correlation coefficient $(r)$ was 0.690 , suggesting a moderate positive correlation.

The association between extraction time and the difficulty indices are shown in Table 2. The mean extraction times according to difficulty index were $12.18 \pm 5.39,18.28 \pm 5.34$, and 28.20 \pm 5.17 minutes in I, II, and III, respectively. The correlation between extraction time and difficulty index was significant $(P<0.001)$, and the observed correlation coefficient $(\mathrm{r})$ was 0.584 , suggesting a moderate positive correlation.(Fig. 3) Extraction time was significantly different between difficulty index grades I and II $(P=0.001)$, I and III $(P<0.001)$, and II and III $(P<0.001)$. (Table 2)

The mean extraction times according to HDI were
$10.60 \pm 4.33,16.30 \pm 7.33,15.38 \pm 4.06,24.08 \pm 3.23,31.60$, and $27.36 \pm 0.33$ minutes in one, one-half, two, two-half, three, and three-half, respectively, and those according to ODI were $10.60 \pm 4.33,15.44 \pm 4.28,24.38 \pm 3.49$, and $27.36 \pm 5.33$ minutes in I, II, III, and IV. The correlations between extraction time and HDI and ODI were significant $(P<0.001)$. The observed correlation coefficients (r) were 0.728 and 0.764 , respectively, suggesting strong positive correlations.(Fig. 4)

Extraction time significantly differed among HDI grades $(P<0.001)$, but post hoc analysis showed significance only between HDI grades two and two-half $(P<0.001)$. Extraction time was significantly different among the ODI grades, ODI grades I and II $(P=0.010)$, II and III $(P<0.001)$, and II and IV

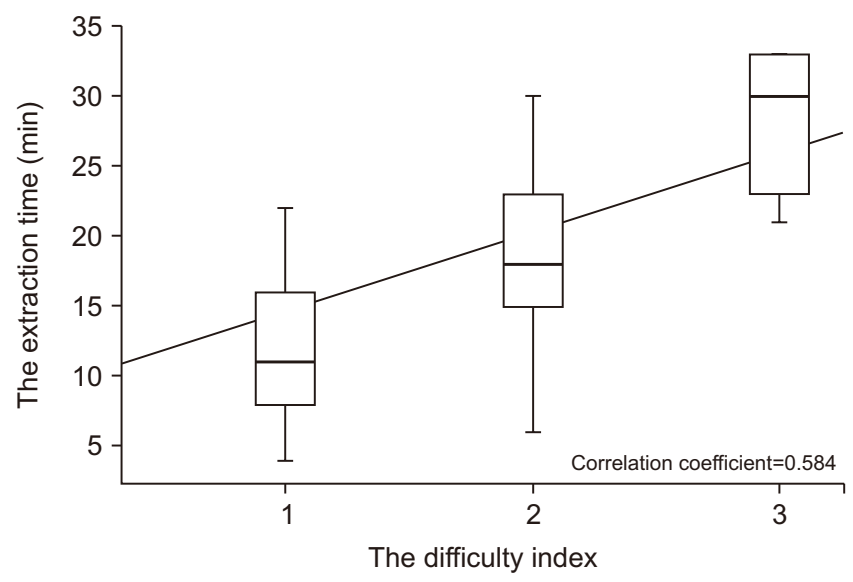

Fig. 3. The correlation between the extraction time and the difficulty index. The correlation coefficient was 0.584 , suggesting a moderate positive correlation $(P<0.001)$.

Jeong-Kui Ku et al: Development and validation of a difficulty index for mandibular third molars with extraction time. J Korean Assoc Oral Maxillofac Surg 2020

Table 2. Association between extraction time and difficulty indices of impacted third molars

\begin{tabular}{|c|c|c|c|c|c|}
\hline Grade & $\mathrm{n}$ & Extraction time (min) & $P$-value & & Correlation coefficient $(r)$ \\
\hline \multicolumn{6}{|c|}{ Difficulty index } \\
\hline I & 17 & $12.18 \pm 5.39$ & \multirow[t]{3}{*}{$<0.001^{1}$} & $0.001^{2}$ (I vs II) & \multirow[t]{3}{*}{0.599} \\
\hline II & 45 & $18.28 \pm 5.34$ & & $<0.001^{2}$ (II vs III) & \\
\hline III & 6 & $28.20 \pm 5.17$ & & & \\
\hline \multicolumn{6}{|c|}{ Half-up difficulty index } \\
\hline 1 & 13 & $10.60 \pm 4.33$ & \multirow{6}{*}{$<0.001^{1}$} & $0.318^{2}(1$ vs 1.5$)$ & \multirow{6}{*}{0.728} \\
\hline 1.5 & 4 & $16.30 \pm 7.33$ & & $>0.999^{2}(1.5$ vs 2$)$ & \\
\hline 2 & 30 & $15.38 \pm 4.06$ & & $<0.001^{2}(2$ vs 2.5$)$ & \\
\hline 2.5 & 15 & $24.08 \pm 3.23$ & & $>0.999^{2}(2.5$ vs 3.5$)$ & \\
\hline 3 & 1 & 31.60 & & NA (2.5 vs 3$)$ & \\
\hline 3.5 & 5 & $27.36 \pm 0.33$ & & NA (3 vs 3.5$)$ & \\
\hline \multicolumn{6}{|c|}{ One-up difficult index } \\
\hline I & 13 & $10.60 \pm 4.33$ & \multirow[t]{4}{*}{$<0.001^{1}$} & $0.010^{2}$ (I vs II $)$ & \multirow[t]{4}{*}{0.764} \\
\hline II & 34 & $15.44 \pm 4.28$ & & $<0.001^{2}$ (II vs III) & \\
\hline III & 16 & $24.38 \pm 3.49$ & & $>0.999^{2}$ (III vs IV) & \\
\hline IV & 5 & $27.36 \pm 5.33$ & & & \\
\hline
\end{tabular}

(NA: not available)

${ }^{1}$ One-way ANOVA. ${ }^{2}$ Post hoc test with Bonferroni correction.

Values are presented as mean \pm standard deviation.

Jeong-Kui Ku et al: Development and validation of a difficulty index for mandibular third molars with extraction time. J Korean Assoc Oral Maxillofac Surg 2020 
A

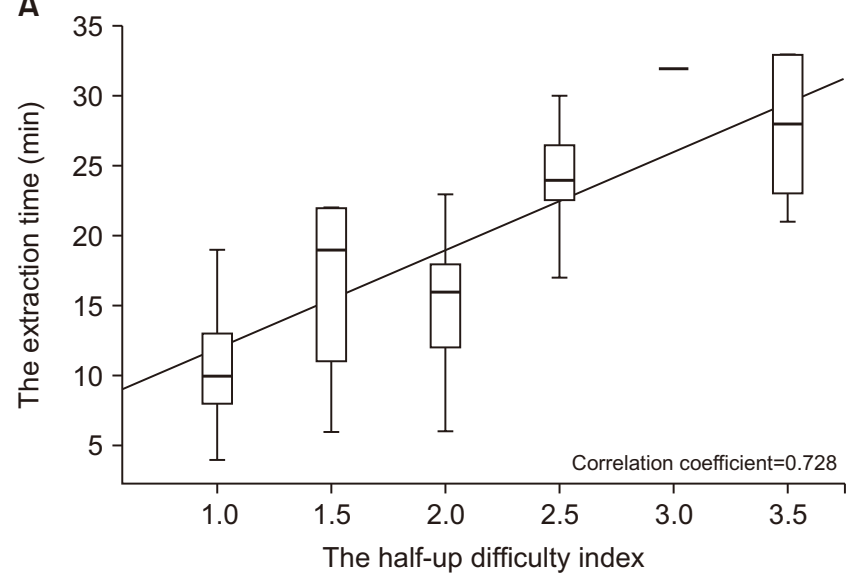

B

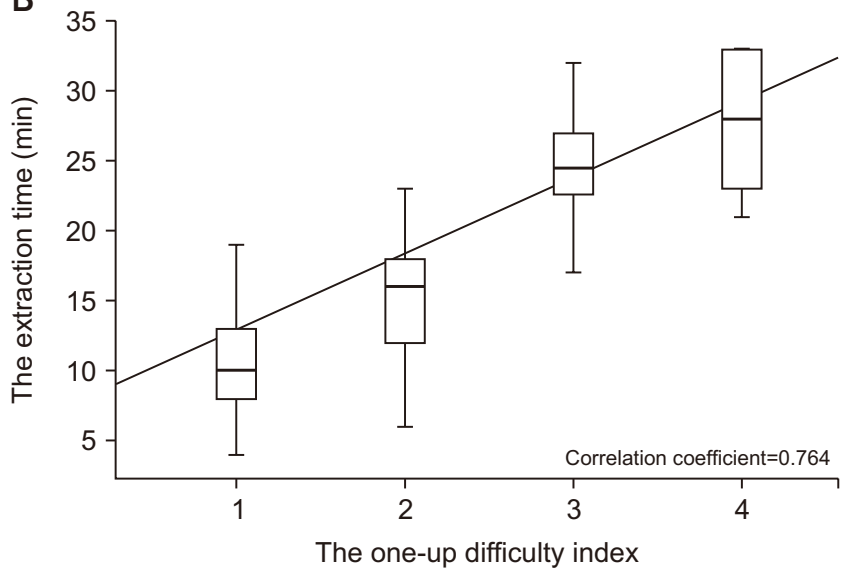

Fig. 4. The correlation between the extraction time and the modified difficulty indices. A. The correlation between the extraction time and the half-up difficulty index. The correlation coefficient was 0.728 , suggesting a strong positive correlation $(P<0.001)$. B. The correlation between the extraction time and the half-up difficulty index. The correlation coefficient was 0.764 , suggesting a strong positive correlation $(P<0.001)$.

Jeong-Kui Ku et al: Development and validation of a difficulty index for mandibular third molars with extraction time. J Korean Assoc Oral Maxillofac Surg 2020

$(P<0.001)$.(Table 2)

\section{Discussion}

Two findings were revealed in this study. First, the difficulty index ${ }^{5}$ classified as grades I, II, and III based on CT data reformatted to a panoramic view was correlated with extraction time of impacted third molars other than grade IV. Second, the modified difficulty indices that considered the presence of pathologic conditions were strongly correlated with extraction time.

Prediction of surgical extraction difficulty is important for anticipating postoperative issues and planning treatment options such as general anesthesia in a controlled environment to prevent complications ${ }^{12,13}$. Extraction difficulties were evaluated based on position of the third molars using panoramic radiography ${ }^{1-5}$. The position was associated with postoperative complications such as alveolar nerve injury ${ }^{6}$ and dry socket ${ }^{7}$. However, assessing the difficulty index with panoramic imaging has been controversial due to morphological variations and superimposition of adjacent structures ${ }^{9,14}$. A major concern in three-dimensional analysis using CT is poor reproducibility in setting the three-dimensional axis and selecting a single image $\mathrm{cut}^{15}$. The author evaluated the position with CT reformatted panoramic images. The correlation coefficients (r) of extraction time according to the recently proposed difficulty scores and index were 0.599 and 0.584 , respectively, on the panoramic image ${ }^{5}$, moderate correlations $(P<0.001)$.
Many studies on extraction of impacted third molars have focused on postoperative complications ${ }^{6,7,9,11,14}$. However, the presence of the following pathologic conditions associated with third molars could also be critical for intraoperative considerations: unfavorable fracture during odontomy in carious third molars, local anesthetic failure and prolonged bleeding during the management of inflammatory conditions, dentigerous cysts or curved roots, and poor accessibility for instrumentation due to cuspal contact between the third molar and the distal surface of the adjacent second molar. Therefore, we considered the presence of pathologic conditions as an independent factor to evaluate the difficulty of extractions. As a result, the coefficient (r) of extraction time was 0.690 with the modified difficulty score based on the presence of pathologic conditions, which was higher than that of the previous difficulty score and exhibited a strong positive correlation $(P<0.001)$. The coefficients (r) further increased to 0.728 and 0.764 with HDI and ODI, respectively. Regarding the result, pathologic conditions could more strongly correlate with extraction time compared with the previous difficulty score by using radiography only. Furthermore, ODI exhibited a greater correlation with surgery time than did HDI.

The inter-grade of the previous difficulty index showed statistically significant differences in average extraction time, but the difference was almost twice as large between grades II and III (18.28 and 28.20 minutes; $P<0.001$ ) compared to the difference between grades I and II (12.18 and $18.28 \mathrm{~min}-$ utes; $P=0.001)$. However, the average extraction time of ODI seems to be subdivided between ODI grades I and II (10.60 
and 15.44 minutes; $P=0.010$ ), II and III (15.44 and 24.38 minutes; $P<0.001$ ), and III and IV (24.38 and 27.36 minutes; $P>0.999)$ compared with the previous difficulty index. ODI grade IV did not exhibit a statistical difference from ODI grade III in Bonferroni's method, a finding that may be explained by the limitation of our retrospective study design that did not include a large number of high-grade extractions.

Surgery time is crucial for patients and clinicians and allows them to determine the difficulty of extraction. As impacted third molars and their surrounding structures vary in anatomy, various surgical techniques have been used. However, few studies have evaluated difficulties based on surgery time. Although we used a unified surgical technique (operculectomy), the operculum could develop after resorption of the bone overlying the eruption space. As a result, we could not include extremely difficult third molars (difficulty index IV) that were fully embedded in bone ${ }^{5}$. Impacted third molars with difficulty index IV have different histologic aspects compared to other impacted teeth and can be removed with alternative treatment strategies. Further studies with prospective designs and larger numbers of patients are needed to evaluate extremely difficult third molars associated with pathologic conditions.

\section{Conclusion}

Extraction time of third molar surgery is strongly correlated with modified difficulty indices that take into consideration the pathologic condition compared to the previous difficulty index. With regard to the clinical situation, the difficulty index of surgical extractions should include an assessment of the pathologic conditions associated with third molars.

\section{ORCID}

Jeong-Kui Ku, https://orcid.org/0000-0003-1192-7066

Na-Hee Chang, https://orcid.org/0000-0001-8634-571X

Yeong-Kon Jeong, https://orcid.org/0000-0002-8768-6150

Sung Hyun Baik, https://orcid.org/0000-0003-3738-8988

Sun-Kyu Choi, https://orcid.org/0000-0001-8827-7060

\section{Authors' Contributions}

J.K.K., N.H.C., and Y.K.J. were responsible for the conception and design of the study and drafting of the article. S.K.C. was responsible for statistical analysis of data, and the statistical methods and results. Y.K.J. was responsible for critical revision of the article. N.H.C., S.H.B., and J.K.K. were responsible for the acquisition of the data, laboratory or clinical/literature search and analysis, and interpretation of the data collected. All authors read and approved the final manuscript.

\section{Ethics Approval and Consent to Participate}

The study was approved by the Institutional Review Board (IRB) at Armed Forces Capital Hospital (No. AFCH-19IRB-034). This retrospective study has received a waiver of written informed consent after review by the IRB.

\section{Conflict of Interest}

No potential conflict of interest relevant to this article was reported.

\section{References}

1. Pell GJ, Gregory GT. Impacted mandibular third molars: classification and modified technique for removal. Dent Dig 1933;39:330-7.

2. Yuasa H, Kawai T, Sugiura M. Classification of surgical difficulty in extracting impacted third molars. Br J Oral Maxillofac Surg 2002;40:26-31. https://doi.org/10.1054/bjom.2001.0684

3. Gbotolorun OM, Arotiba GT, Ladeinde AL. Assessment of factors associated with surgical difficulty in impacted mandibular third molar extraction. J Oral Maxillofac Surg 2007;65:1977-83. https:// doi.org/10.1016/j.joms.2006.11.030

4. Sammartino G, Gasparro R, Marenzi G, Trosino O, Mariniello M, Riccitiello F. Extraction of mandibular third molars: proposal of a new scale of difficulty. Br J Oral Maxillofac Surg 2017;55:952-7. https://doi.org/10.1016/j.bjoms.2017.09.012

5. Kim JY, Yong HS, Park KH, Huh JK. Modified difficult index adding extremely difficult for fully impacted mandibular third molar extraction. J Korean Assoc Oral Maxillofac Surg 2019;45:309-15. https://doi.org/10.5125/jkaoms.2019.45.6.309

6. Ishii S, Abe S, Moro A, Yokomizo N, Kobayashi Y. The horizontal inclination angle is associated with the risk of inferior alveolar nerve injury during the extraction of mandibular third molars. Int J Oral Maxillofac Surg 2017;46:1626-34. https://doi.org/10.1016/ j.ijom.2017.07.010

7. Eshghpour M, Nejat AH. Dry socket following surgical removal of impacted third molar in an Iranian population: incidence and risk factors. Niger J Clin Pract 2013;16:496-500. https://doi. org/10.4103/1119-3077.116897

8. Shin YS. How to improve the national health insurance fee-forservice schedule. Health Welf Policy Forum 2019;272:39-52.

9. Kugelberg CF, Ahlström U, Ericson S, Hugoson A. Periodontal healing after impacted lower third molar surgery. A retrospective study. Int J Oral Surg 1985;14:29-40. https://doi.org/10.1016/ s0300-9785(85)80007-7

10. Moloney J, Stassen LF. Pericoronitis: treatment and a clinical dilemma. J Ir Dent Assoc 2009:55:190-2.

11. Chaves AJ, Nascimento LR, Costa ME, Franz-Montan M, Oliveira-Júnior PA, Groppo FC. Effects of surgical removal of mandibular third molar on the periodontium of the second molar. Int J Dent Hyg 2008;6:123-8. https://doi.org/10.1111/j.1601- 
5037.2008.00297.x

12. Khan M, Mehmboob B, Katpar S. Extraction of wisdom teeth under general anesthesia- a study. J Khyber Coll Dent 2014;5:20-4.

13. Savanheimo N, Sundberg SA, Virtanen JI, Vehkalahti MM. Dental care and treatments provided under general anaesthesia in the Helsinki Public Dental Service. BMC Oral Health 2012;12:45. https:// doi.org/10.1186/1472-6831-12-45

14. Sigron GR, Pourmand PP, Mache B, Stadlinger B, Locher MC. The most common complications after wisdom-tooth removal: part 1: a retrospective study of 1,199 cases in the mandible. Swiss Dent J 2014;124:1042-6, 1052-6.

15. Cevidanes LH, Styner MA, Proffit WR. Image analysis and superimposition of 3-dimensional cone-beam computed tomography models. Am J Orthod Dentofacial Orthop 2006;129:611-8. https:// doi.org/10.1016/j.ajodo.2005.12.008

How to cite this article: $\mathrm{Ku} \mathrm{JK}$, Chang $\mathrm{NH}$, Jeong YK, Baik SH, Choi SK. Development and validation of a difficulty index for mandibular third molars with extraction time. J Korean Assoc Oral Maxillofac Surg 2020;46:328-334. https://doi.org/10.5125/jkaoms.2020.46.5.328 